\title{
THE EFFECT OF YOLK SAC ABSORPTION ON THE GROWTH OF SMALL INTESTINAL VILLI IN BROILER CHICKS WITH HEALED AND UNHEALED NAVEL
}

\author{
GHAITH HAMMADEH; MUSTAFA ALHALLAK and MUWAFFAQ JUNAID \\ Department of Anatomy, Faculty of Veterinary Medicine, Hama University, Syria, Hama
}

Received: 18 August 2016; Accepted: 29 September 2016

\begin{abstract}
By the time of hatching, the residual yolk sac (RYS) should be completely internalized into the body cavity of the chick through the navel, then the navel should be completely closed (healed). After hatching, the RYS contents are used for general growth including growth of the small intestine. The aim of this study was to verify if there is any effect of navel condition on the growth of small intestinal villi. Jejunum samples from 200 new hatched chicks (at the age of 1 up to 5 days), with the average of 20 chicks per day were obtained, and divided into 2 groups: healed navels and unhealed navels, which were collected and studied histologically. For the first time, this study demonstrated the negative effect of unhealed navel on the growth of small intestinal villi. Morphometric Parameters Measurements of small intestinal also demonstrated villi that the height and the surface areas of villi were increased in healed navel chicks in comparison with unhealed chicks.
\end{abstract}

Key words: Chicks, Intestinal villi, Navel, Yolk sac

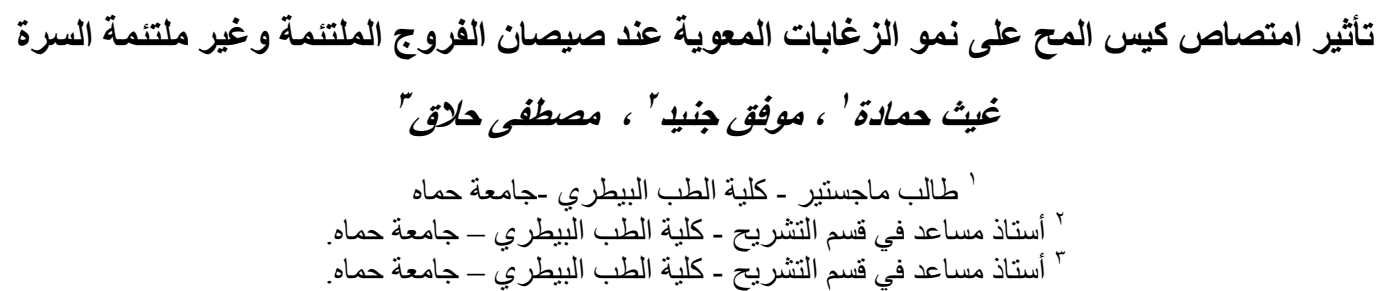

مع اقتر اب مو عد الفقس يدخل كيس المح المتبقي تجويف البطن عبر سرة الصوص ثم تلتئم السرة بشكل كامل. توظف محتويـات كبس

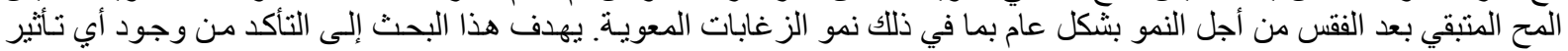

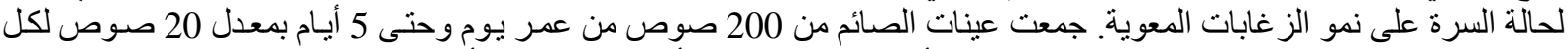

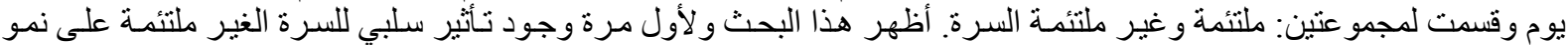

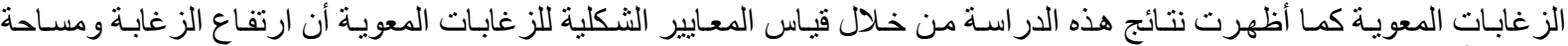
سطحها أكبر في الصيصان الملتئمة السرة مقارنة بالصيصان الغير ملتئمة السرة. الكلمات المفتاحية: الصيصـان- الزغابات المعوية ـ السرة ـ كيس المح المقدمــة

\section{INTRODUCTOIN}

تمتد عملية فقس البيض في المفاقس التجارية عادة الى حوالي 48 ساعة بعد 19 يوماً من التحضين. أصبحت المفرخات التجارية في

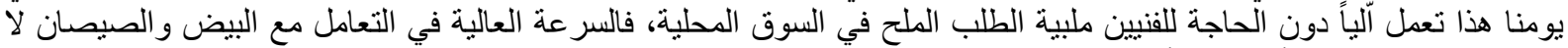

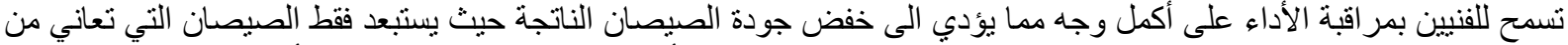

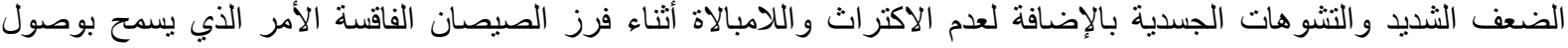
صيصان منخفضة الجودة لمزارع التونية التربية.

يعاني العديد من المربين من ظاهرة عدم التئام السرة (قثرة ندبية دموية فوق السرة) في الصيصان الفاقسة حديثاً و التي لا تلاحظ

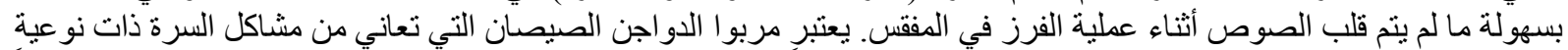

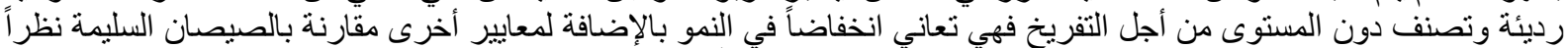

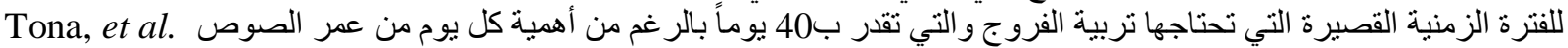

.(2004)

Corresponding author: Dr. GHAITH HAMMADEH

E-mail address: ghaith9841@gmail.com

Present address: Department of Anatomy, Faculty of Veterinary Medicine, Hama University, Syria, Hama 


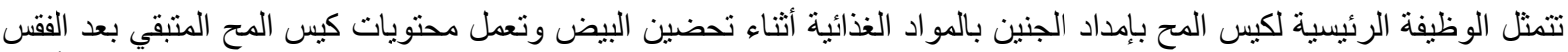

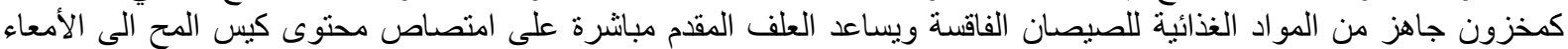

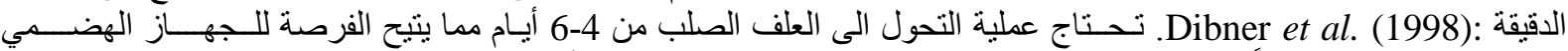

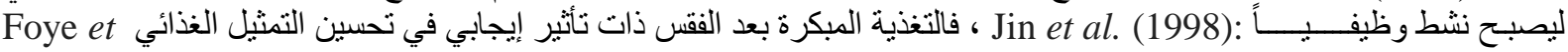

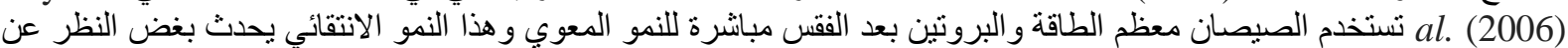

وجود الغذاء أو عدمه (1999) Noy and sklan.

يدخل كيس المح تجويف بطن الصوص في اليوم 19 من التحضين عبر السرة وفي الظروف المثالية عند الفقس يرتد كيس المح وينتني

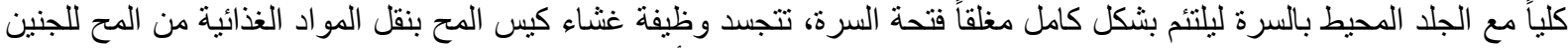

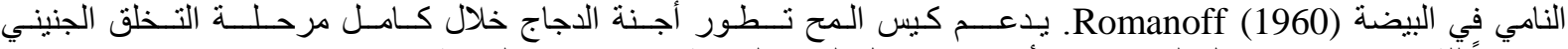

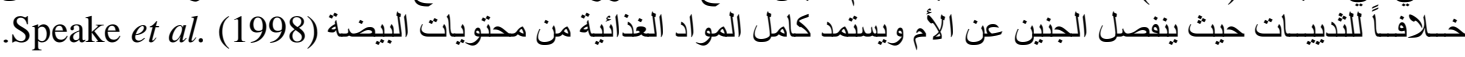

امتصاص المح يؤمن نمو ونضوج القناة المعدية المعوية وبالتالي يضمن نمو أفضل بعد الفقس، تُظهر الوظائف الفيسيولوجية

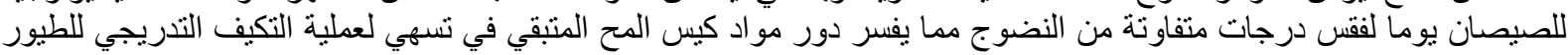

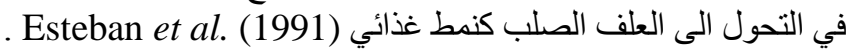

هدف البحث Objective of The Study:

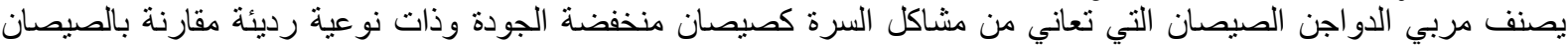

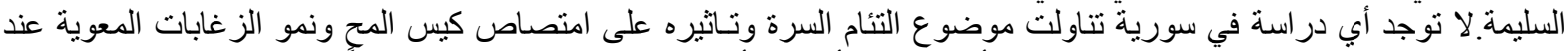

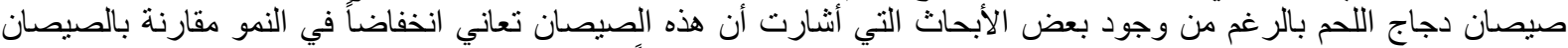

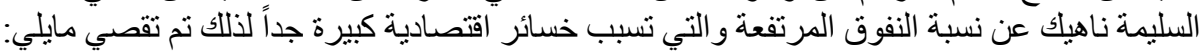

ا. . التغير ات الثكلية لكيس المح المتبقي (وزن كيس المح ونسبة امتصاصه).

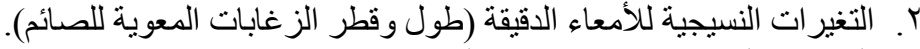

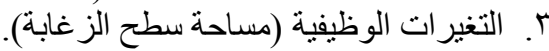

\section{المواد وطر ائق العمل \\ MATERIALS AND METHODS}

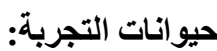
أجريت الدراسة على الجى 200 صوص من دجاج اللحم من سلالة روص (Ross) 308 و التي قسمت لمجمو عتين: المجموعة الأولى

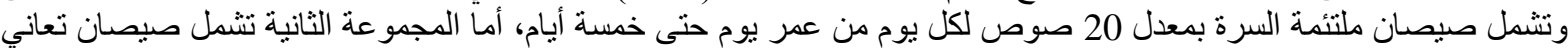

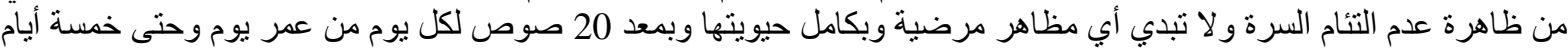

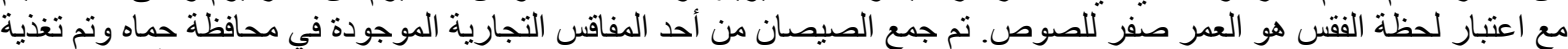
الصيصان في كلا المجمو عتين على خلطة علفية خاصنة بدجاج اللحم مرحلة أولى وتمت معاملة العينات ودر آستها نسيجياً.

جدول رقم ا : المكونات الغذائية للخلطة العلفية مرحلة أولى:

\begin{tabular}{|c|c|}
\hline خلطة علفية مرحلة أولى & المادة العلفية \% \\
\hline $0 Y .00$ & ذرة صفر اء \% \\
\hline r9.0 & كسبة صويا \% \\
\hline$\varepsilon$ & زيت صويا \% \\
\hline$r$ & فوسفات ثنائية الكالسيوم \% \\
\hline .90 & كربونات الكالسيوم \% \\
\hline$\cdot r$ & ميثيونين حر \% \\
\hline$\because 1$ & كلوريد الكولين \% \\
\hline .1 & خلطة فيتامينات \% \\
\hline .1 & خلطة معادن نادرة \% \\
\hline.$\leqslant 0$ & ملح طعام \% \\
\hline$\because 0$ & مضاد كو كسيديا \% \\
\hline
\end{tabular}




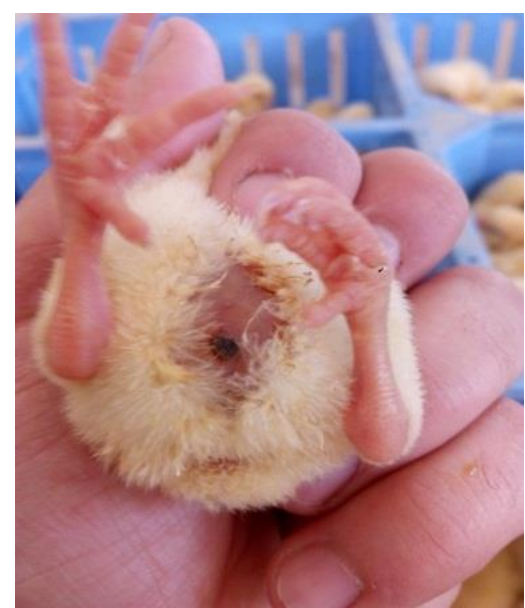

شكل رقم ب : يبين: صيصان غير ملتئمة السرة

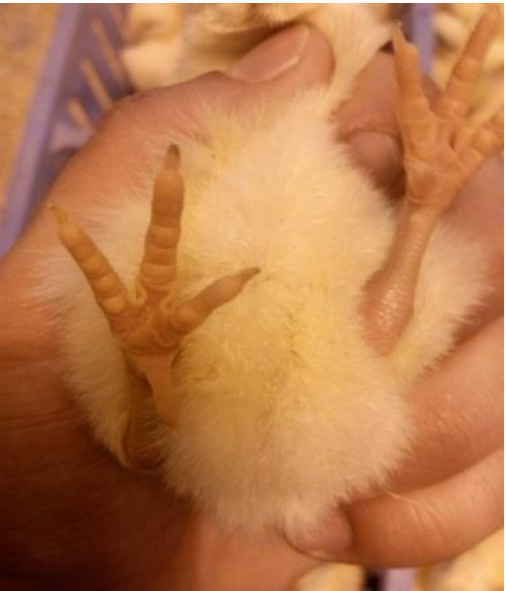

شكل رقم ا : يبين: صيصان ملتئمة السرة

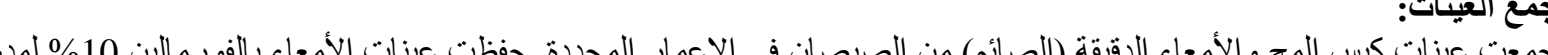

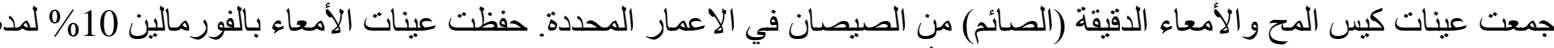

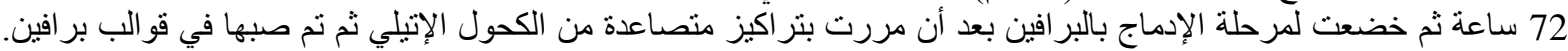

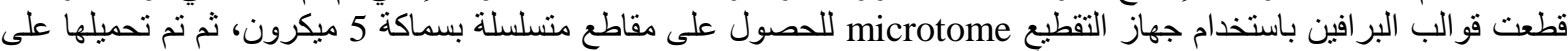
شر ائح زجاجية وصبغت بالهيماتوكسيلين والأيوزين (H\&E).

وزن كيس المح:

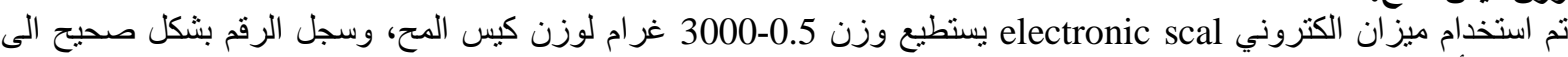
الغر ام الأقرب الظاهر على على شئشة الميزان.

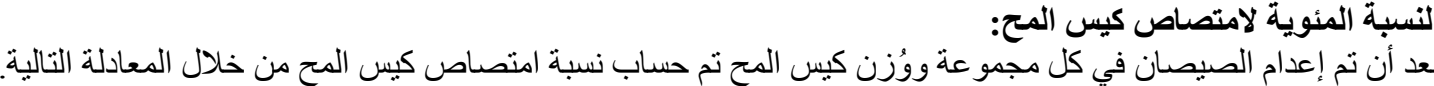
نسبة امتصاص كيس المح خلان المرحلة $100 x$ وزن كيس المح في بداية المرحلة - وزن كيس المح في نهاية المرحلة وزن كيس المح في بداية المرحلة

قياس المؤشرات الشكلية: تم قياس المعايير المختلفة باستئعمال أداة القياس العينية الدقيقة Micrometer Eye Piece. وأجريت القياسات المختلفة من طول

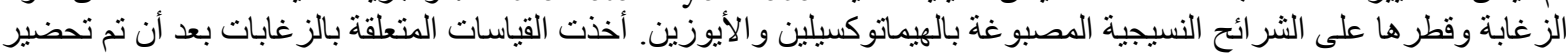
شر ائح نسيجية عدد 1من كل قالب (عينة)، وتم قياس 3 ز غابات بشكل عشو ائي من من كل شريحة زئي زاجية.

$$
\text { حساب طول الزغابة: تم قياس المسافة بين قمة الزغابة وقاعدتها. }
$$

حساب مساحة سطح الزغابة: يتم حساب مساحة سطح الزغابة الذي يعبر عن مساحة امتصاصها من خلال التقريب لعلم الهندسة

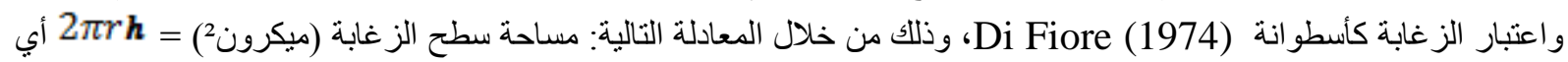

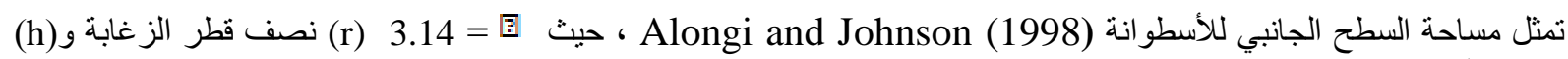
ارتفاع الزغابة.

التحليل الاحصائى:

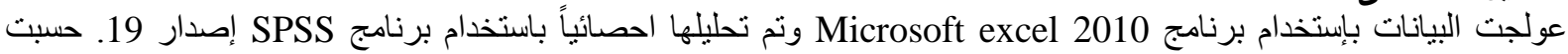

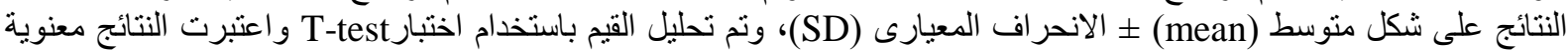
عندما تكون 0.05 > 


\section{النتائسمج}

\section{RESULTS}

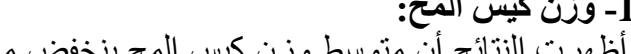

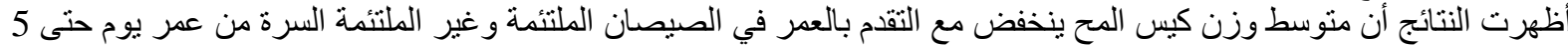

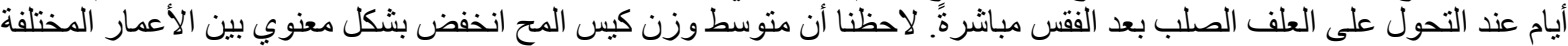

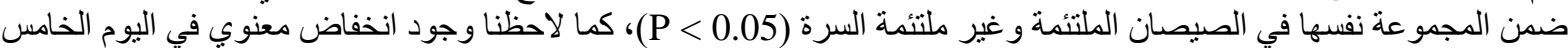

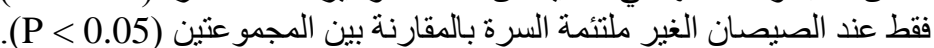

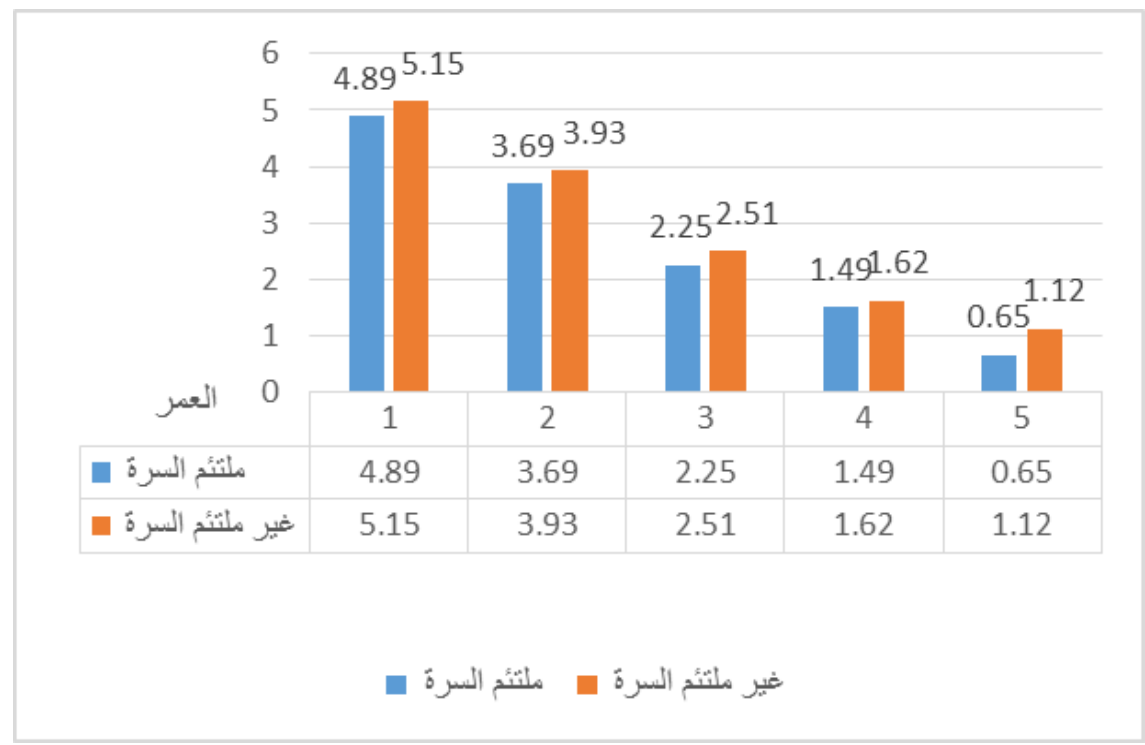

مخطط بياني رقم 1: وزن كيس المح (غ)

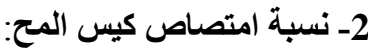

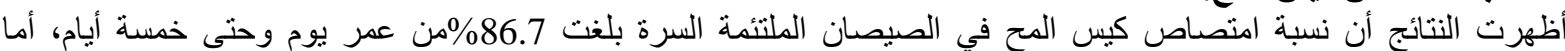

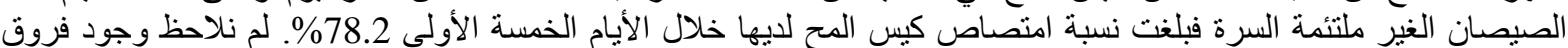
معنوية في نسبة امتصاص كيس المح بالمقارنة بين المجمو عتين (P > (P)

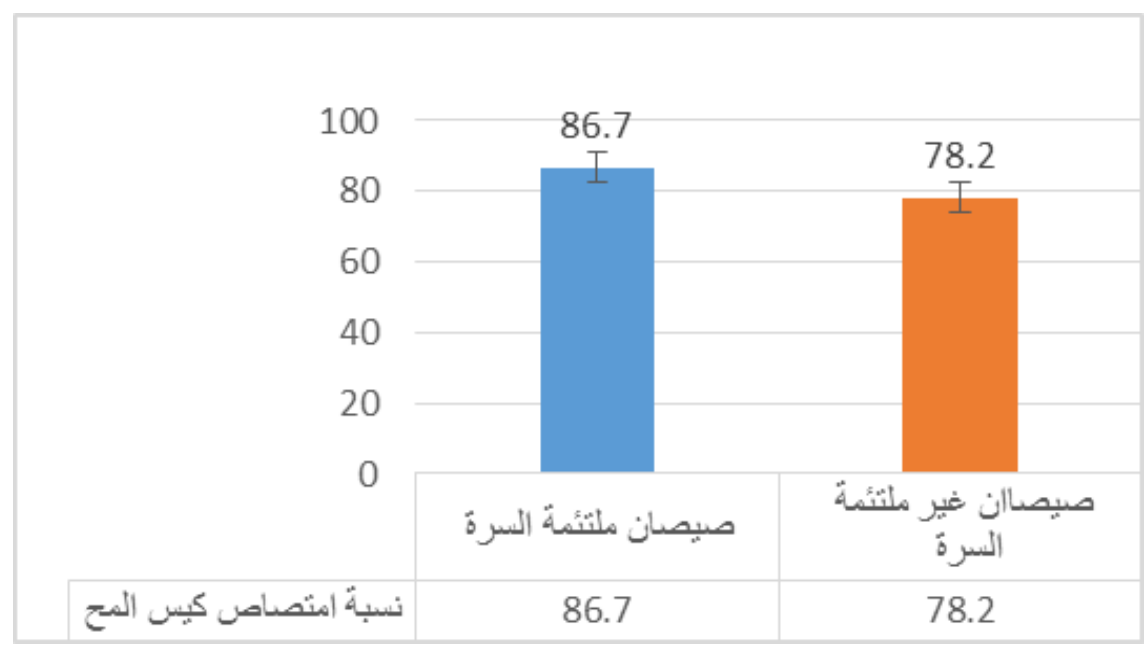

مخطط بياني رقم 2: نسبة امتصاص كيس المح \%

3- 3 - 3ول الزغابة.

أظهرت النتائج أن متوسط طولة الزول الزغابة يزداد مع التقدم بالـعر وعند التحول على العلف الصلب كمصدر للغذاء في كلا

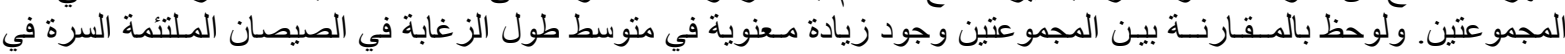
ليوم 2و4و5 حيث (P (P) 


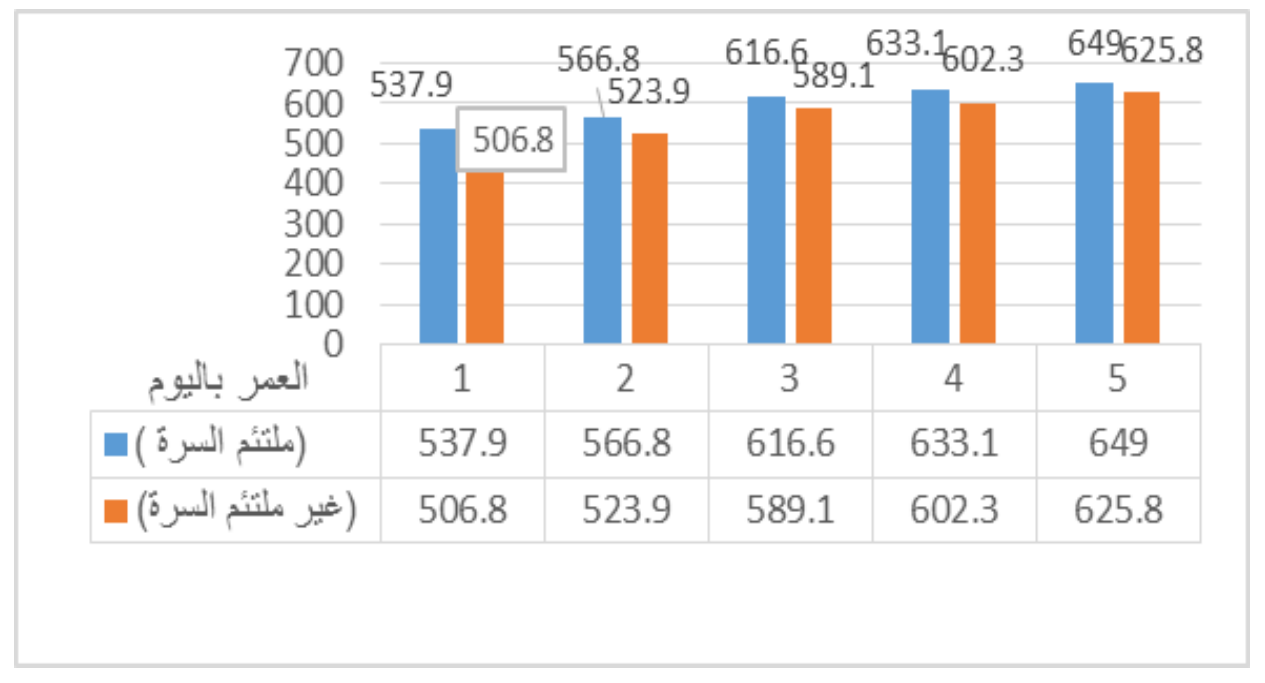

مخطط بياني رقم 3: طول الزغابة (ميكرومتر )
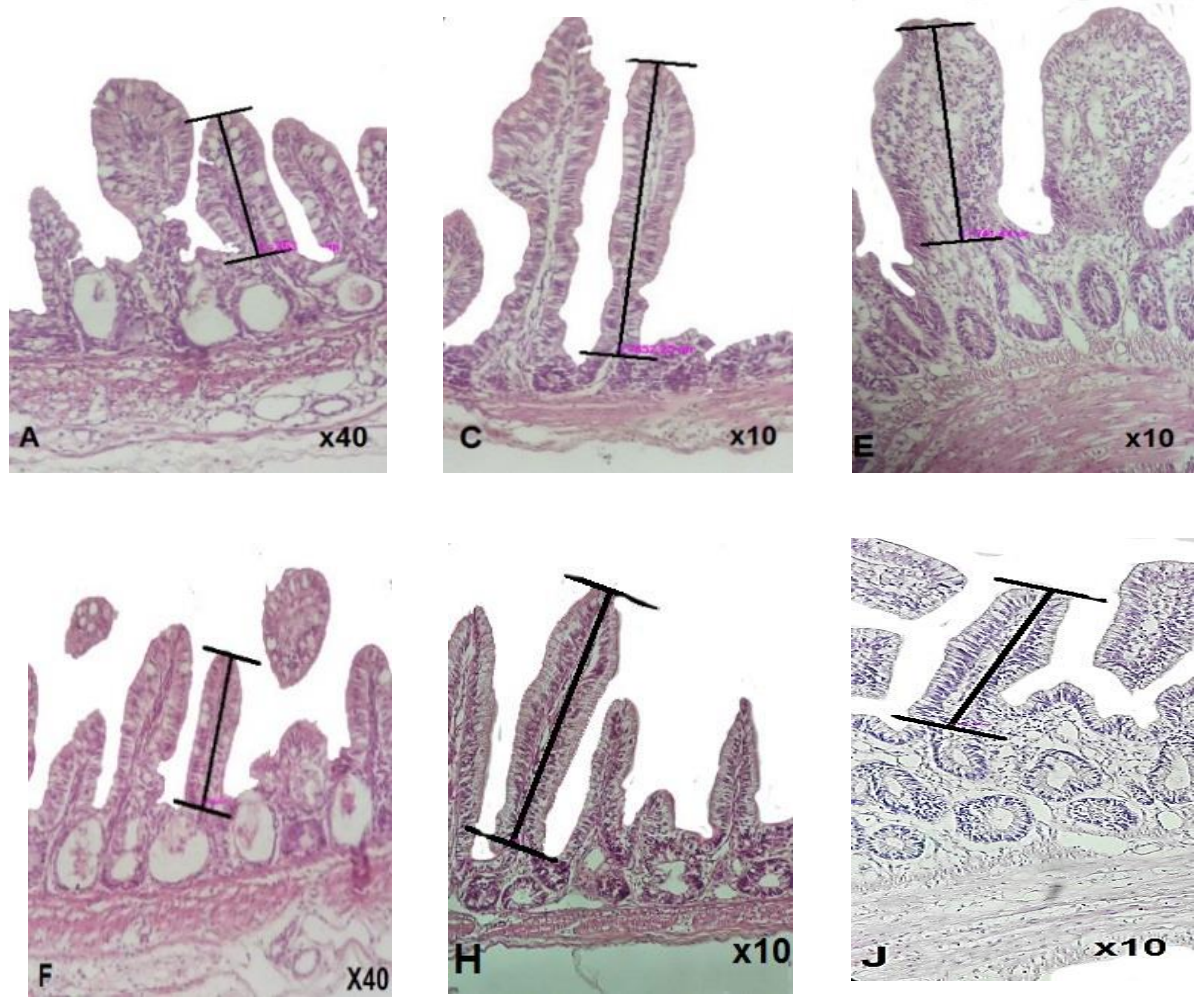

\section{Day1}

Day3

Day5

شكل رقم (3): طول الزغابة في اليوم (13،3) في الصيصان الملتئمة السرة (في الأعلى) وغير ملتئمة السرة (في الأسفل).

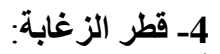

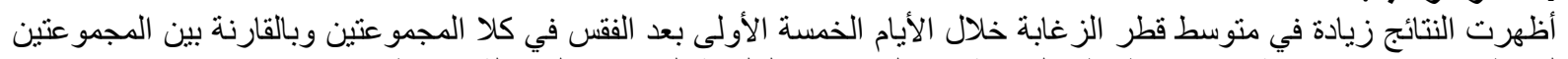

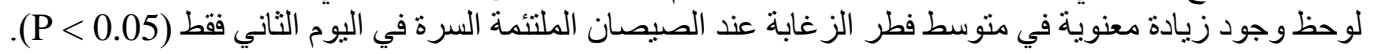




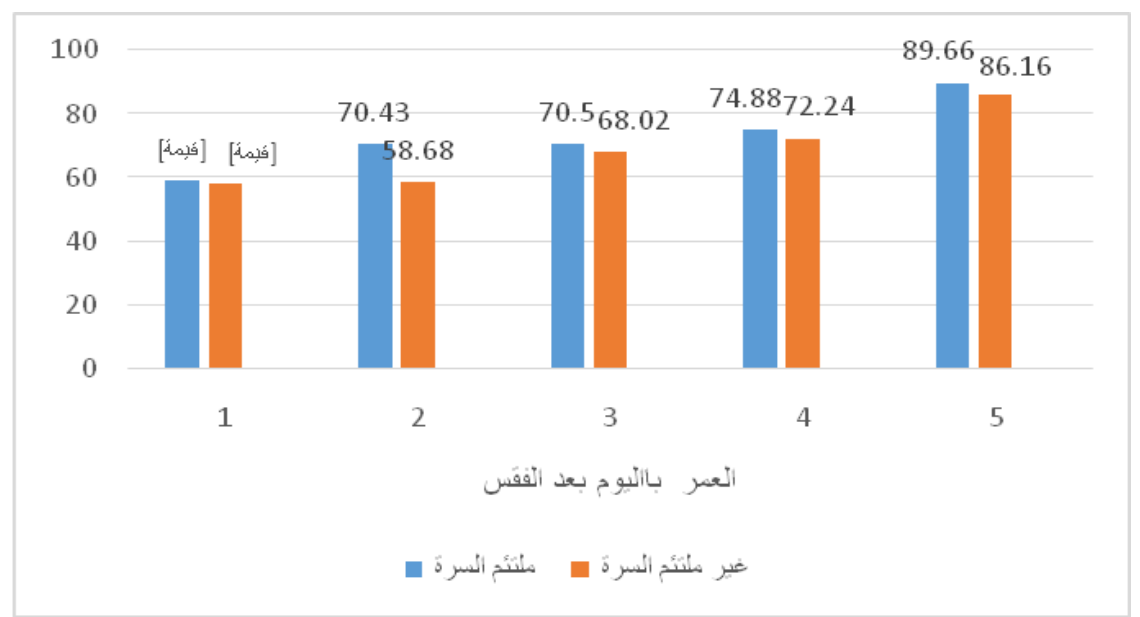

مخطط بياني رقم 4: منوسط قطر الزغابة بعد الفقس (ميكرون)
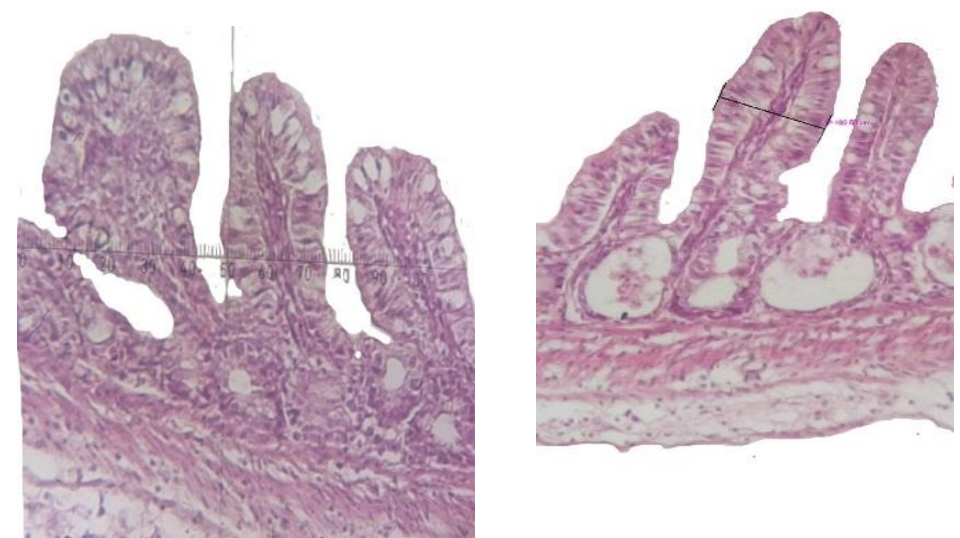

شكل رقم (4): طريقة قياس قطر الزغابة باستخدام العدسة العينية (عاليمين)، قطر الزغابة بعمر يومين عند الصيصان الملتئمة السرة عاليسار (قوة تكبير 100). (عالينين)

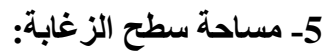

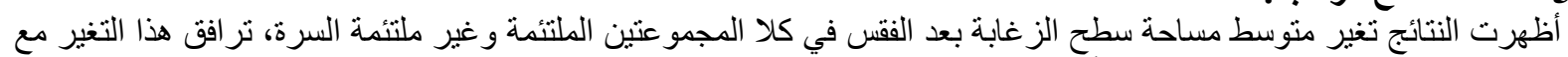

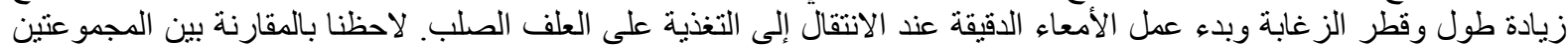

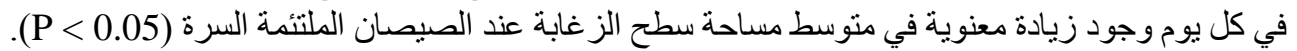

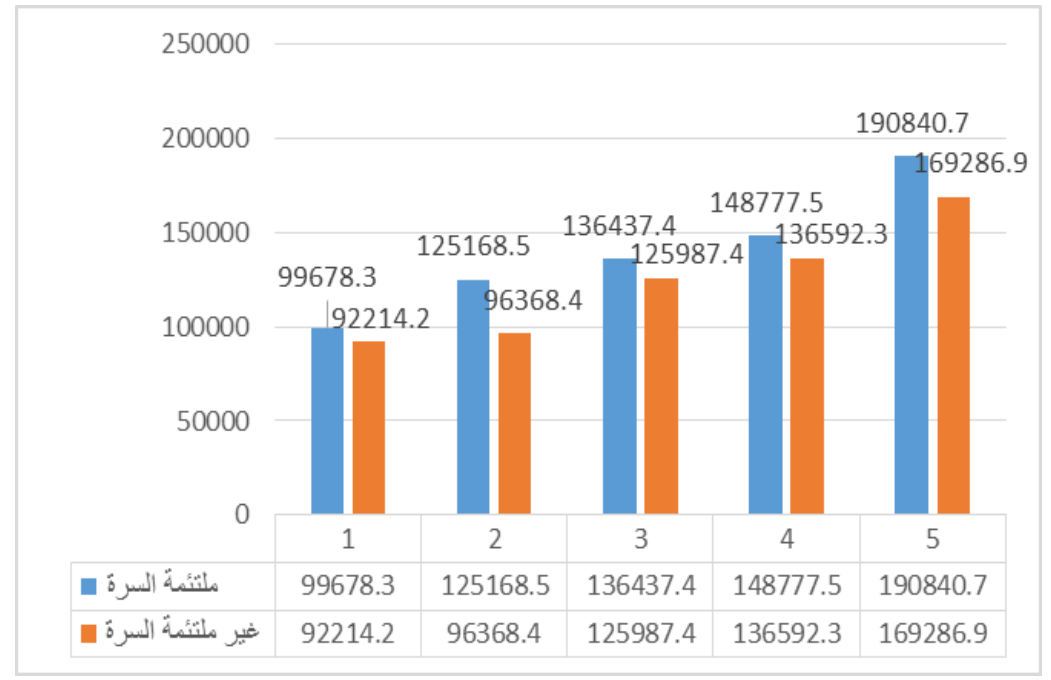

مخطط بياني رقمه : مساحة سطح الزغابة بعد الفقس (ميكرون2) 


\section{المناقشّة \\ DISCUSSION}

هذا البحث هو أول دراسة تجرى في سورية تتتاول تأثير امتصاص كيس المح على تطور الأمعاء الدقيقة عند الصيصان التي تعاني

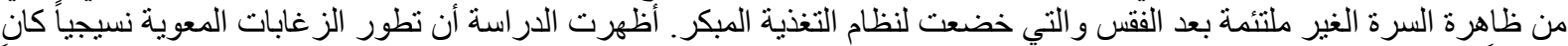

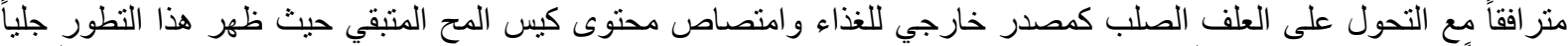

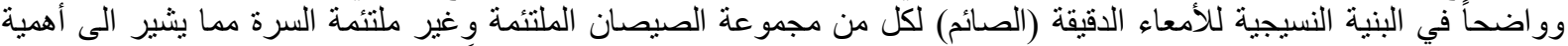

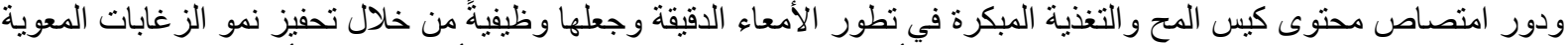

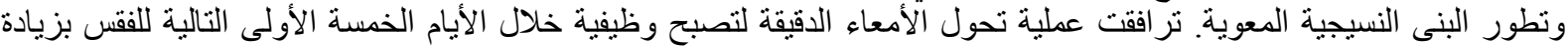

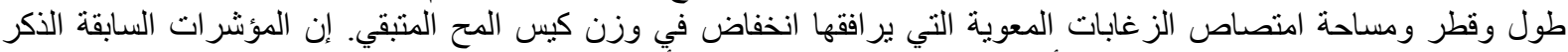

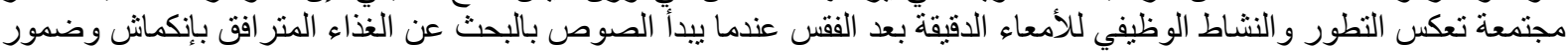
حجم كيس المح.

يمثل كيس المح عند الفقس المصدر الرئيسي للمو اد الغذائية للصوص الفاقس حتى ينعرف الصوص على بئيتّه الجديدة و يبدأ تنتاول

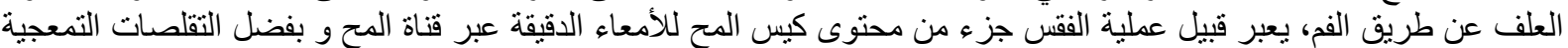

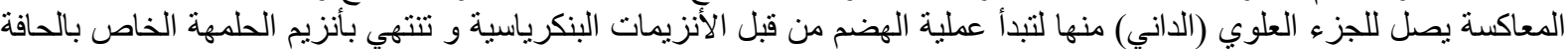

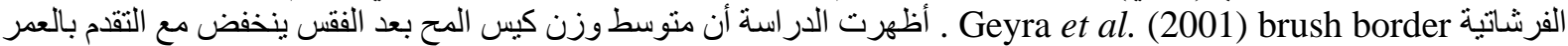

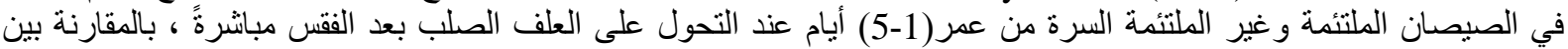

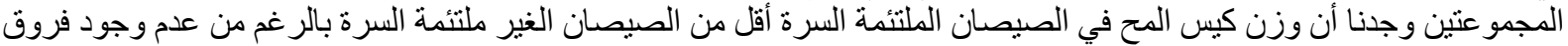

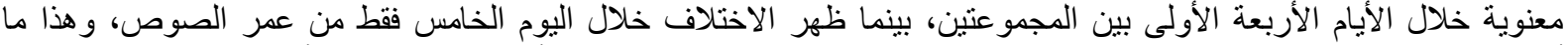

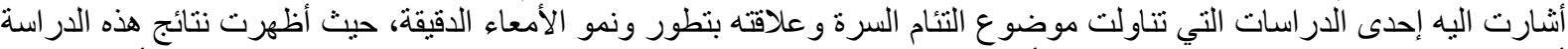

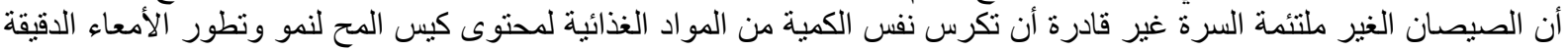

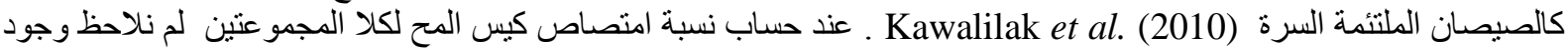

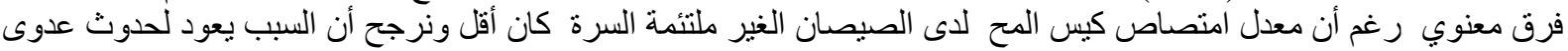

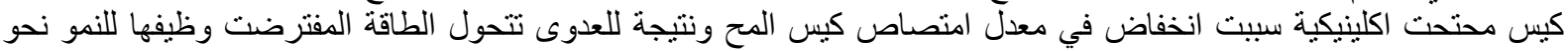

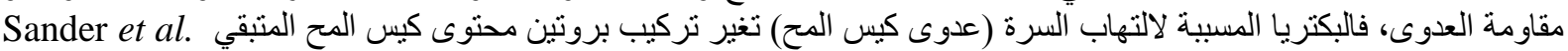

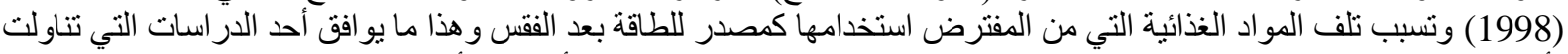

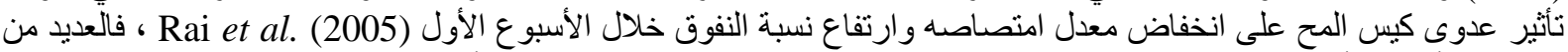

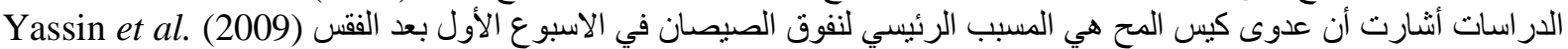

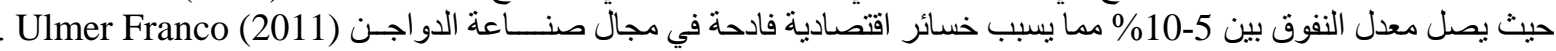

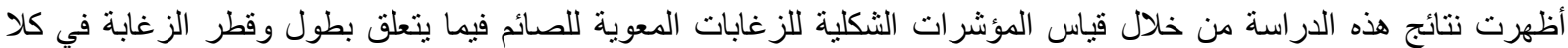

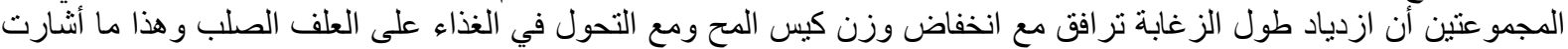

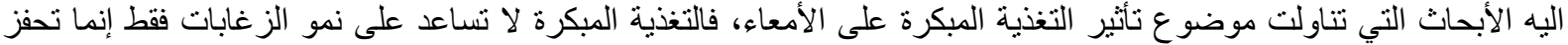

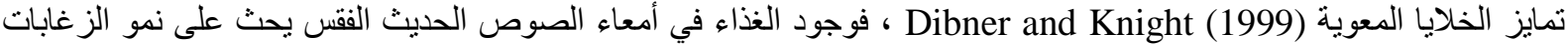

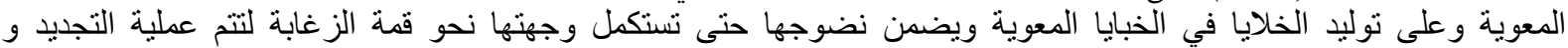

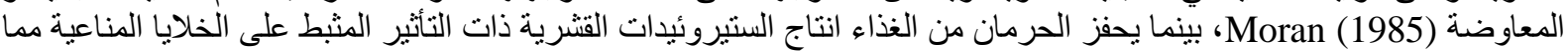

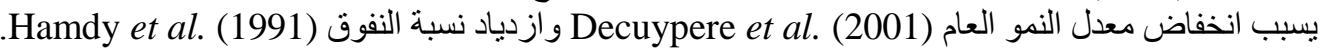

لاحظنا عند مقارنة طول الز غابة بين المجو عتين وجود زيادة معنوي في اليوم 2، 4، 5 حيث كانت زغابات الصيصان الملتنئمة السرة أطول ولم نلاحظ وجود فرق معنوي في قطر الزغابة سين سوا في اليوم الثناني.

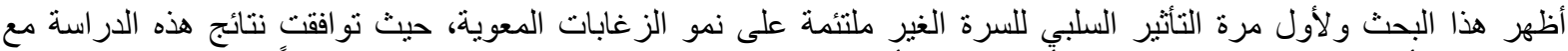

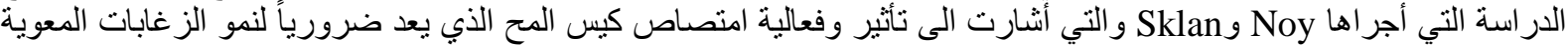

.Noy and Sklan (1999)

أشارت العديد من الدراسات السابقة أن ارتفاع الزغابات المعوية ومساحة سطحها ينخفضان كلما اتجهنا من الجزء الداء الداني للأمعاء

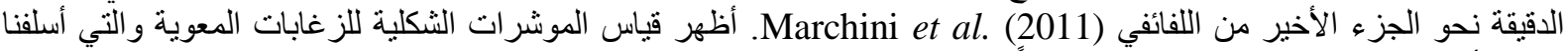

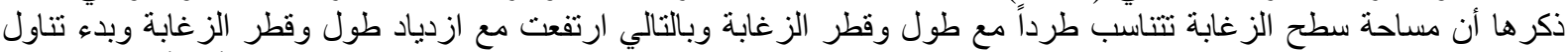

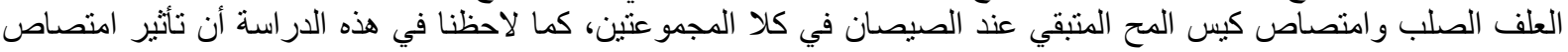

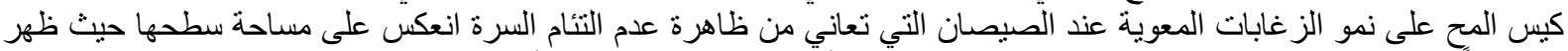

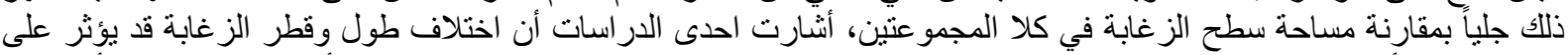

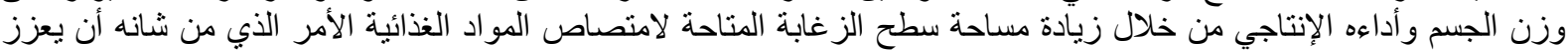
Schaefer et al. النمو و الحصبلة الانتاجية (2006) 


\section{الاستنتاجات \\ CONCLUSIONS}

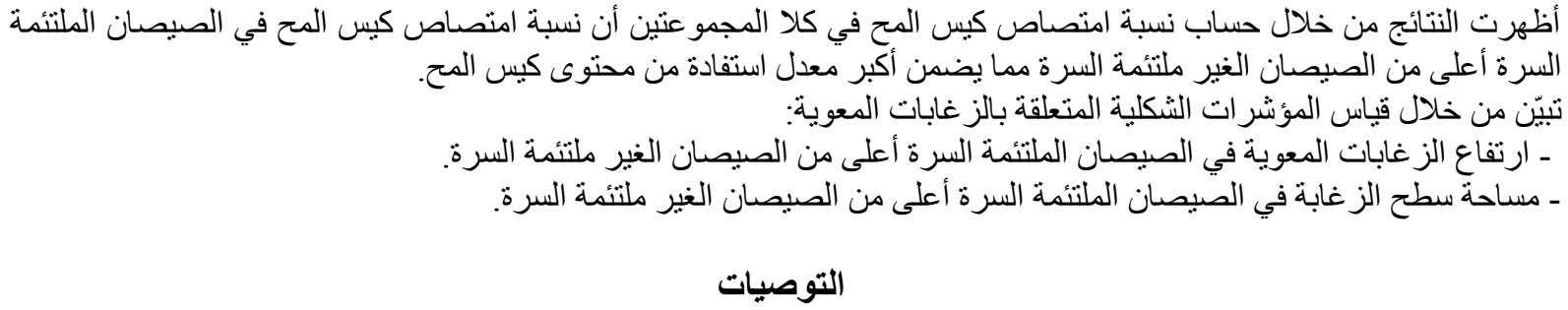

\section{RECOMMENDATIONS}

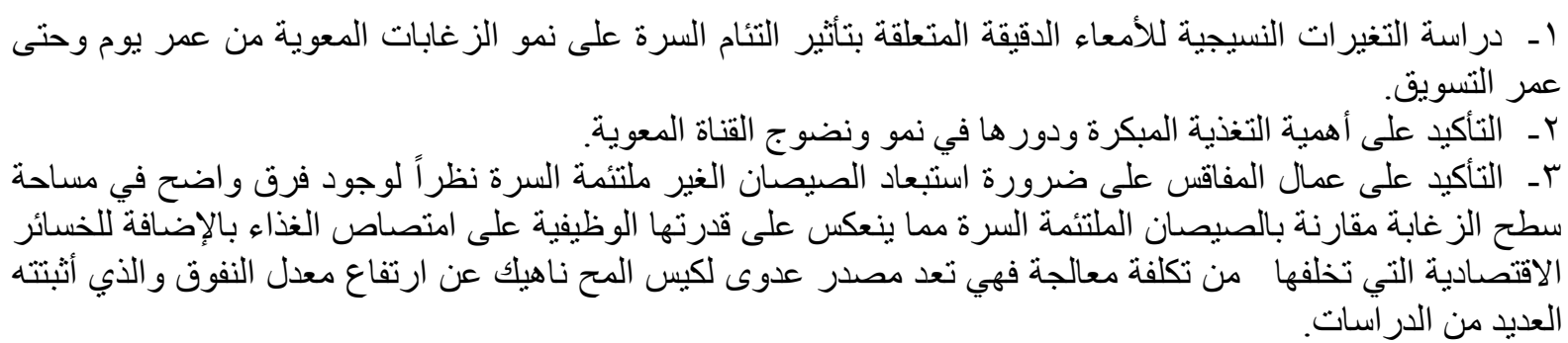

\section{REFERENCES}

Alongi, D. and Johnson, P. (1998): Digestive Landscapes Exploring surface area in the intestine. A \& P TECHNOLOGIST, pp: 175-184

Decuypere, E.; Tona, K.; Bruggeman, V. and Bamelis, E. (2001): The day-old chick: a crucial hinge between breeders and broilers. World's Poultry Sci J. 57:127-138.

Dibner, J.J. and Knight, C.D. (1999): Early feeding and gut health in hatchlings. Int. Hatchery Practice 14(1). Positive Action Publications, Ltd., Middlesex, NJ.

Dibner, J.; Knight, C.D.; Kitchell, M.; Atwell, C.; Downs, A. and Ivey, F.J. (1998): Early feeding and development of the immune system in neonatal poultry. J. Appl. Poult. Res, 7: 425-436.

Di Fiore, M. (1974): Atlas of Human Histology, Philadelphia: Lea \& Febiger, $4^{\text {th }}$ Edition.

Esteban, S.; Rayo, M.J.; Moreno, M.; Sastre, M.; Rial, R.V. and Tur, J.A. (1991): A Role played by thevitelline diverticulum in the yolk sac resorption in young post-hatched chickens. J. Comp. Physiol, B, 160: 645-648.

Foye, O.T.; Uni, Z.; McMurtry, J.P. and Ferket, P.R. (2006): The effects of amniotic nutrient administration, "in ovo feeding" of arginine and/or $\beta$-hydroxy- $\beta$-methyl butyrate (HMB) on insulin-like growth factors, energy metabolism and growth in turkey poults. Int. J. Poult. Sci. 5: 309-317.

Geyra, A.; Uni, Z. and Sklan, D. (2001): Enterocyte dynamics and mucosal development in the posthatch chick. Poult. Sci, 80:776-782.

Hamdy, AM.; Henken, AM.; Van Der Wel, H.; Galal, AG. and Abdelmoty, AK. (1991): Effects of incubation humidity and hatching time on heat tolerance of neonatal chicks: growth performance after heat exposure. Poultry Sci. 70:1507-1515.

Jin, S.-H.; Corless, A. and Sell, J.L. (1998): Digestive system development in post hatch poultry. World's Poult. Sci. J. 54:335-345.

Kawalilak, L.T.; Ulmer Franco, A.M. and Fasenko, G.M. (2010): Impaired intestinal villi growth in broiler chicks with unhealed navels. Poultry Science 89:82-87.

Marchini, C.F.; Silva, P.L.; Nascimento, M.R.; Beletti, M.E.; Silva, N.M. and Guimarães, E.C. (2011): Body weight, intestinal morphometry and cell proliferation of broiler chickens submitted to cyclic heat stress. Int. J. Poultry Sci., 10: 455-460.

Moran, E.T. (1985): Digestion and absorption of carbohydrates in fowl and events through perinatal development. J. Nutr., 115: 665-674.

Noy, Y. and Sklan, D. (1999): Energy utilization in newly hatched chicks. Poult. Sci, 78:1750-1756. 
Rai, M.F.; Khan, S.A.; Aslam, A. and Khalid, S. (2005): Effects of yolk sac infection in chicken.Avian Poult. Biol. Rev. 16:87-93.

Romanoff, A.L. (1960): The extraembryonic membranes. In the avianembryo: structural and functional development. The Macmillan Company, New York, pp: 1041-1140.

Schaefer, C.M.; Corsiglia, C.M.; Mireles, A. and Koutsos, E.A. (2006): Turkey breeder hen age affects growth and systemic and intestinal inflammatory responses in female poults examined at different ages post-hatch. Poultry Sci., 85: 1755-1763.

Sander, J.E.; Willinghan, E.M.; Wilson, J.L. and Thayers, S.G. (1998): The effect of inoculating Enterococcus faecalis into the yolk sac on chick quality and maternal antibody absorption. Avian Dis. 42:359-363.

Speake, B.K.; Murray, A.M.B. and Noble, C.R. (1998): Transport and transformations of yolk lipids during development of the avian embryo. Prog. Lipid Res, 37(1): 1-32.

Tona, K.; Onagbesan, O.M.; Jego, Y.; Kamers, B.; Decuypere, E. and Bruggeman, V. (2004): Comparison of embryo physiological parameters during incubation, chick quality, and growth performance of three lines of broiler breeders differing in genetic composition and growth rate. Poult. Sci., 83: 507-513.

Ulmer Franco, A.M. (2011): Yolk Sac Infections in Broiler Chicks: Studies on Escherichia coli, Chick Acquired Immunity and Barn Microbiology. PhD thesis, University of Alberta Edmonton, Alberta, pp: 1-197.

Yassin, H.; Velthuis, A.G.J.; Boerjan, M. and Van Rielt, J. (2009): Field study on broilers' first-week mortality. Poult. Sci., 88: 798-804. 